\title{
Search for Lepton-Flavor Violation in the Decay $\tau^{-} \rightarrow \ell^{-} \ell^{+} \ell^{-}$
}

B. Aubert,${ }^{1}$ R. Barate,${ }^{1}$ D. Boutigny, ${ }^{1}$ F. Couderc,${ }^{1}$ J.-M. Gaillard, ${ }^{1}$ A. Hicheur, ${ }^{1}$ Y. Karyotakis,${ }^{1}$ J. P. Lees,${ }^{1}$ V. Tisserand, ${ }^{1}$ A. Zghiche, ${ }^{1}$ A. Palano, ${ }^{2}$ A. Pompili, ${ }^{2}$ J. C. Chen,${ }^{3}$ N. D. Qi,${ }^{3}$ G. Rong, ${ }^{3}$ P. Wang, ${ }^{3}$ Y. S. Zhu, ${ }^{3}$ G. Eigen,${ }^{4}$ I. Ofte, ${ }^{4}$ B. Stugu,${ }^{4}$ G. S. Abrams,${ }^{5}$ A. W. Borgland,${ }^{5}$ A. B. Breon,${ }^{5}$ D. N. Brown, ${ }^{5}$ J. Button-Shafer,${ }^{5}$

R. N. Cahn, ${ }^{5}$ E. Charles, ${ }^{5}$ C. T. Day,${ }^{5}$ M. S. Gill, ${ }^{5}$ A. V. Gritsan,${ }^{5}$ Y. Groysman,${ }^{5}$ R. G. Jacobsen,${ }^{5}$

R. W. Kadel,${ }^{5}$ J. Kadyk,${ }^{5}$ L. T. Kerth, ${ }^{5}$ Yu. G. Kolomensky, ${ }^{5}$ G. Kukartsev, ${ }^{5}$ C. LeClerc, ${ }^{5}$ M. E. Levi, ${ }^{5}$ G. Lynch,${ }^{5}$ L. M. Mir,${ }^{5}$ P. J. Oddone,${ }^{5}$ T. J. Orimoto, ${ }^{5}$ M. Pripstein,${ }^{5}$ N. A. Roe, ${ }^{5}$ M. T. Ronan, ${ }^{5}$ V. G. Shelkov,${ }^{5}$

A. V. Telnov, ${ }^{5}$ W. A. Wenzel, ${ }^{5}$ K. Ford,${ }^{6}$ T. J. Harrison, ${ }^{6}$ C. M. Hawkes,${ }^{6}$ S. E. Morgan,${ }^{6}$ A. T. Watson, ${ }^{6}$

N. K. Watson, ${ }^{6}$ M. Fritsch, ${ }^{7}$ K. Goetzen, ${ }^{7}$ T. Held,${ }^{7}$ H. Koch, ${ }^{7}$ B. Lewandowski, ${ }^{7}$ M. Pelizaeus,${ }^{7}$ M. Steinke, ${ }^{7}$ J. T. Boyd,$^{8}$ N. Chevalier,${ }^{8}$ W. N. Cottingham, ${ }^{8}$ M. P. Kelly, ${ }^{8}$ T. E. Latham,${ }^{8}$ F. F. Wilson, ${ }^{8}$ K. Abe, ${ }^{9}$

T. Cuhadar-Donszelmann, ${ }^{9}$ C. Hearty,${ }^{9}$ T. S. Mattison, ${ }^{9}$ J. A. McKenna, ${ }^{9}$ D. Thiessen, ${ }^{9}$ P. Kyberd,${ }^{10}$

L. Teodorescu, ${ }^{10}$ V. E. Blinov, ${ }^{11}$ A. D. Bukin, ${ }^{11}$ V. P. Druzhinin,,${ }^{11}$ V. B. Golubev ${ }^{11}$ V. N. Ivanchenko,${ }^{11}$

E. A. Kravchenko, ${ }^{11}$ A. P. Onuchin, ${ }^{11}$ S. I. Serednyakov, ${ }^{11}$ Yu. I. Skovpen, ${ }^{11}$ E. P. Solodov,${ }^{11}$ A. N. Yushkov ${ }^{11}$

D. Best,${ }^{12}$ M. Bruinsma, ${ }^{12}$ M. Chao, ${ }^{12}$ I. Eschrich, ${ }^{12}$ D. Kirkby,${ }^{12}$ A. J. Lankford, ${ }^{12}$ M. Mandelkern, ${ }^{12}$

R. K. Mommsen, ${ }^{12}$ W. Roethel,${ }^{12}$ D. P. Stoker,${ }^{12}$ C. Buchanan, ${ }^{13}$ B. L. Hartfiel,${ }^{13}$ J. W. Gary, ${ }^{14}$ B. C. Shen, ${ }^{14}$

K. Wang,${ }^{14}$ D. del Re ${ }^{15}$ H. K. Hadavand,${ }^{15}$ E. J. Hill,${ }^{15}$ D. B. MacFarlane, ${ }^{15}$ H. P. Paar,${ }^{15}$ Sh. Rahatlou,${ }^{15}$

V. Sharma, ${ }^{15}$ J. W. Berryhill, ${ }^{16}$ C. Campagnari, ${ }^{16}$ B. Dahmes, ${ }^{16}$ S. L. Levy,${ }^{16}$ O. Long, ${ }^{16}$ A. Lu, ${ }^{16}$ M. A. Mazur, ${ }^{16}$ J. D. Richman, ${ }^{16}$ W. Verkerke, ${ }^{16}$ T. W. Beck,,${ }^{17}$ A. M. Eisner, ${ }^{17}$ C. A. Heusch,,${ }^{17}$ W. S. Lockman, ${ }^{17}$ T. Schalk, ${ }^{17}$ R. E. Schmitz, ${ }^{17}$ B. A. Schumm, ${ }^{17}$ A. Seiden, ${ }^{17}$ P. Spradlin, ${ }^{17}$ D. C. Williams,,${ }^{17}$ M. G. Wilson, ${ }^{17}$ J. Albert, ${ }^{18}$ E. Chen, ${ }^{18}$ G. P. Dubois-Felsmann, ${ }^{18}$ A. Dvoretskii, ${ }^{18}$ D. G. Hitlin, ${ }^{18}$ I. Narsky, ${ }^{18}$ T. Piatenko, ${ }^{18}$ F. C. Porter,${ }^{18}$

A. Ryd, ${ }^{18}$ A. Samuel, ${ }^{18}$ S. Yang, ${ }^{18}$ S. Jayatilleke, ${ }^{19}$ G. Mancinelli, ${ }^{19}$ B. T. Meadows, ${ }^{19}$ M. D. Sokoloff, ${ }^{19}$

T. Abe,${ }^{20}$ F. Blanc,${ }^{20}$ P. Bloom,${ }^{20}$ S. Chen,${ }^{20}$ P. J. Clark, ${ }^{20}$ W. T. Ford, ${ }^{20}$ U. Nauenberg, ${ }^{20}$ A. Olivas,${ }^{20}$ P. Rankin, ${ }^{20}$ J. G. Smith, ${ }^{20}$ W. C. van Hoek,${ }^{20}$ L. Zhang, ${ }^{20}$ J. L. Harton, ${ }^{21}$ T. Hu, ${ }^{21}$ A. Soffer, ${ }^{21}$ W. H. Toki, ${ }^{21}$ R. J. Wilson, ${ }^{21}$ D. Altenburg, ${ }^{22}$ T. Brandt,,${ }^{22}$ J. Brose,${ }^{22}$ T. Colberg, ${ }^{22}$ M. Dickopp, ${ }^{22}$ E. Feltresi, ${ }^{22}$ A. Hauke, ${ }^{22}$ H. M. Lacker, ${ }^{22}$ E. Maly, ${ }^{22}$ R. Müller-Pfefferkorn,${ }^{22}$ R. Nogowski,${ }^{22}$ S. Otto,${ }^{22}$ J. Schubert,${ }^{22}$ K. R. Schubert,${ }^{22}$ R. Schwierz, ${ }^{22}$ B. Spaan, ${ }^{22}$ D. Bernard,${ }^{23}$ G. R. Bonneaud,${ }^{23}$ F. Brochard, ${ }^{23}$ P. Grenier, ${ }^{23}$ Ch. Thiebaux,${ }^{23}$ G. Vasileiadis, ${ }^{23}$ M. Verderi, ${ }^{23}$ D. J. Bard,${ }^{24}$ A. Khan, ${ }^{24}$ D. Lavin, ${ }^{24}$ F. Muheim,${ }^{24}$ S. Playfer, ${ }^{24}$ M. Andreotti ${ }^{25}$ V. Azzolini, ${ }^{25}$ D. Bettoni, ${ }^{25}$ C. Bozzi, ${ }^{25}$ R. Calabrese,${ }^{25}$ G. Cibinetto, ${ }^{25}$ E. Luppi, ${ }^{25}$ M. Negrini,${ }^{25}$ A. Sarti, ${ }^{25}$ E. Treadwell,${ }^{26}$ R. Baldini-Ferroli, ${ }^{27}$ A. Calcaterra,${ }^{27}$ R. de Sangro,${ }^{27}$ G. Finocchiaro, ${ }^{27}$ P. Patteri, ${ }^{27}$ M. Piccolo, ${ }^{27}$ A. Zallo, ${ }^{27}$ A. Buzzo,${ }^{28}$ R. Capra,,${ }^{28}$ R. Contri, ${ }^{28}$ G. Crosetti, ${ }^{28}$ M. Lo Vetere,${ }^{28}$ M. Macri, ${ }^{28}$ M. R. Monge,${ }^{28}$ S. Passaggio, ${ }^{28}$ C. Patrignani ${ }^{28}$ E. Robutti, ${ }^{28}$ A. Santroni, ${ }^{28}$ S. Tosi, ${ }^{28}$ S. Bailey, ${ }^{29}$ G. Brandenburg, ${ }^{29}$ M. Morii, ${ }^{29}$ E. Won, ${ }^{29}$ R. S. Dubitzky, ${ }^{30}$ U. Langenegger, ${ }^{30}$ W. Bhimji, ${ }^{31}$ D. A. Bowerman,${ }^{31}$ P. D. Dauncey ${ }^{31}$ U. Egede,${ }^{31}$ J. R. Gaillard, ${ }^{31}$ G. W. Morton, ${ }^{31}$ J. A. Nash, ${ }^{31}$ G. P. Taylor, ${ }^{31}$ G. J. Grenier, ${ }^{32}$ S.-J. Lee, ${ }^{32}$ U. Mallik, ${ }^{32}$ J. Cochran, ${ }^{33}$ H. B. Crawley, ${ }^{33}$ J. Lamsa, ${ }^{33}$ W. T. Meyer, ${ }^{33}$ S. Prell, ${ }^{33}$ E. I. Rosenberg, ${ }^{33}$ J. Yi,,${ }^{33}$ M. Davier ${ }^{34}$ G. Grosdidier ${ }^{34}$ A. Höcker, ${ }^{34}$ S. Laplace, ${ }^{34}$ F. Le Diberder, ${ }^{34}$ V. Lepeltier, ${ }^{34}$ A. M. Lutz, ${ }^{34}$ T. C. Petersen, ${ }^{34}$ S. Plaszczynski, ${ }^{34}$ M. H. Schune, ${ }^{34}$ L. Tantot,${ }^{34}$ G. Wormser, ${ }^{34}$ C. H. Cheng, ${ }^{35}$ D. J. Lange, ${ }^{35}$ M. C. Simani,${ }^{35}$ D. M. Wright, ${ }^{35}$ A. J. Bevan, ${ }^{36}$ J. P. Coleman, ${ }^{36}$ J. R. Fry, ${ }^{36}$ E. Gabathuler, ${ }^{36}$ R. Gamet, ${ }^{36}$ M. Kay ${ }^{36}$ R. J. Parry, ${ }^{36}$ D. J. Payne,${ }^{36}$ R. J. Sloane, ${ }^{36}$ C. Touramanis, ${ }^{36}$ J. J. Back,${ }^{37}$ P. F. Harrison, ${ }^{37}$ G. B. Mohanty,${ }^{37}$ C. L. Brown, ${ }^{38}$

G. Cowan, ${ }^{38}$ R. L. Flack, ${ }^{38}$ H. U. Flaecher, ${ }^{38}$ S. George, ${ }^{38}$ M. G. Green, ${ }^{38}$ A. Kurup,${ }^{38}$ C. E. Marker, ${ }^{38}$

T. R. McMahon, ${ }^{38}$ S. Ricciardi, ${ }^{38}$ F. Salvatore, ${ }^{38}$ G. Vaitsas, ${ }^{38}$ M. A. Winter, ${ }^{38}$ D. Brown,${ }^{39}$ C. L. Davis,${ }^{39}$ J. Allison, ${ }^{40}$ N. R. Barlow, ${ }^{40}$ R. J. Barlow,${ }^{40}$ P. A. Hart, ${ }^{40}$ M. C. Hodgkinson, ${ }^{40}$ G. D. Lafferty, ${ }^{40}$ A. J. Lyon, ${ }^{40}$ J. C. Williams, ${ }^{40}$ A. Farbin, ${ }^{41}$ W. D. Hulsbergen, ${ }^{41}$ A. Jawahery, ${ }^{41}$ D. Kovalskyi, ${ }^{41}$ C. K. Lae ${ }^{41}$ V. Lillard, ${ }^{41}$ D. A. Roberts ${ }^{41}$ G. Blaylock, ${ }^{42}$ C. Dallapiccola ${ }^{42}$ K. T. Flood,${ }^{42}$ S. S. Hertzbach ${ }^{42}$ R. Kofler ${ }^{42}$ V. B. Koptchev, ${ }^{42}$ T. B. Moore, ${ }^{42}$ S. Saremi ${ }^{42}$ H. Staengle, ${ }^{42}$ S. Willocq,${ }^{42}$ R. Cowan ${ }^{43}$ G. Sciolla, ${ }^{43}$ F. Taylor, ${ }^{43}$ R. K. Yamamoto, ${ }^{43}$ D. J. J. Mangeol, ${ }^{44}$ P. M. Patel, ${ }^{44}$ S. H. Robertson, ${ }^{44}$ A. Lazzaro, ${ }^{45}$ F. Palombo, ${ }^{45}$ J. M. Bauer, ${ }^{46}$ L. Cremaldi ${ }^{46}$ 
V. Eschenburg, ${ }^{46}$ R. Godang, ${ }^{46}$ R. Kroeger, ${ }^{46}$ J. Reidy, ${ }^{46}$ D. A. Sanders, ${ }^{46}$ D. J. Summers, ${ }^{46}$ H. W. Zhao, ${ }^{46}$ S. Brunet, ${ }^{47}$ D. Côté, ${ }^{47}$ P. Taras, ${ }^{47}$ H. Nicholson, ${ }^{48}$ C. Cartaro, ${ }^{49}$ N. Cavallo, ${ }^{49}$ F. Fabozzi $,{ }^{49}, *$ C. Gatto, ${ }^{49}$ L. Lista, ${ }^{49}$ D. Monorchio, ${ }^{49}$ P. Paolucci, ${ }^{49}$ D. Piccolo, ${ }^{49}$ C. Sciacca, ${ }^{49}$ M. Baak,${ }^{50}$ G. Raven, ${ }^{50}$ L. Wilden, ${ }^{50}$ C. P. Jessop, ${ }^{51}$ J. M. LoSecco, ${ }^{51}$ T. A. Gabriel, ${ }^{52}$ T. Allmendinger, ${ }^{53}$ B. Brau, ${ }^{53}$ K. K. Gan, ${ }^{53}$ K. Honscheid, ${ }^{53}$ D. Hufnagel, ${ }^{53}$ H. Kagan, ${ }^{53}$ R. Kass, ${ }^{53}$ T. Pulliam, ${ }^{53}$ R. Ter-Antonyan, ${ }^{53}$ Q. K. Wong, ${ }^{53}$ J. Brau, ${ }^{54}$ R. Frey, ${ }^{54}$ O. Igonkina, ${ }^{54}$ C. T. Potter,${ }^{54}$ N. B. Sinev,${ }^{54}$ D. Strom, ${ }^{54}$ E. Torrence,${ }^{54}$ F. Colecchia, ${ }^{55}$ A. Dorigo,${ }^{55}$ F. Galeazzi, ${ }^{55}$ M. Margoni, ${ }^{55}$ M. Morandin, ${ }^{55}$ M. Posocco, ${ }^{55}$ M. Rotondo, ${ }^{55}$ F. Simonetto, ${ }^{55}$ R. Stroili, ${ }^{55}$ G. Tiozzo, ${ }^{55}$ C. Voci, ${ }^{55}$

M. Benayoun, ${ }^{56}$ H. Briand, ${ }^{56}$ J. Chauveau, ${ }^{56}$ P. David, ${ }^{56}$ Ch. de la Vaissière, ${ }^{56}$ L. Del Buono, ${ }^{56}$ O. Hamon, ${ }^{56}$ M. J. J. John, ${ }^{56}$ Ph. Leruste, ${ }^{56}$ J. Ocariz, ${ }^{56}$ M. Pivk, ${ }^{56}$ L. Roos, ${ }^{56}$ S. T'Jampens, ${ }^{56}$ G. Therin, ${ }^{56}$ P. F. Manfredi, ${ }^{57}$ V. Re ${ }^{57}$ P. K. Behera, ${ }^{58}$ L. Gladney,${ }^{58}$ Q. H. Guo, ${ }^{58}$ J. Panetta, ${ }^{58}$ F. Anulli, ${ }^{27,}{ }^{59}$ M. Biasini, ${ }^{59}$ I. M. Peruzzi, ${ }^{27,}{ }^{59}$

M. Pioppi, ${ }^{59}$ C. Angelini, ${ }^{60}$ G. Batignani, ${ }^{60}$ S. Bettarini, ${ }^{60}$ M. Bondioli, ${ }^{60}$ F. Bucci, ${ }^{60}$ G. Calderini, ${ }^{60}$

M. Carpinelli, ${ }^{60}$ V. Del Gamba, ${ }^{60}$ F. Forti, ${ }^{60}$ M. A. Giorgi, ${ }^{60}$ A. Lusiani, ${ }^{60}$ G. Marchiori, ${ }^{60}$ F. Martinez-Vidal, ${ }^{60}, \dagger$ M. Morganti, ${ }^{60}$ N. Neri, ${ }^{60}$ E. Paoloni, ${ }^{60}$ M. Rama, ${ }^{60}$ G. Rizzo, ${ }^{60}$ F. Sandrelli, ${ }^{60}$ J. Walsh, ${ }^{60}$ M. Haire, ${ }^{61}$ D. Judd, ${ }^{61}$

K. Paick, ${ }^{61}$ D. E. Wagoner, ${ }^{61}$ N. Danielson, ${ }^{62}$ P. Elmer, ${ }^{62}$ C. Lu, ${ }^{62}$ V. Miftakov, ${ }^{62}$ J. Olsen, ${ }^{62}$ A. J. S. Smith, ${ }^{62}$ E. W. Varnes ${ }^{62}$ F. Bellini, ${ }^{63}$ G. Cavoto, ${ }^{62,}{ }^{63}$ R. Faccini, ${ }^{63}$ F. Ferrarotto, ${ }^{63}$ F. Ferroni, ${ }^{63}$ M. Gaspero, ${ }^{63}$ L. Li Gioi, ${ }^{63}$

M. A. Mazzoni, ${ }^{63}$ S. Morganti, ${ }^{63}$ M. Pierini, ${ }^{63}$ G. Piredda, ${ }^{63}$ F. Safai Tehrani, ${ }^{63}$ C. Voena, ${ }^{63}$ S. Christ, ${ }^{64}$

G. Wagner, ${ }^{64}$ R. Waldi, ${ }^{64}$ T. Adye, ${ }^{65}$ N. De Groot,${ }^{65}$ B. Franek, ${ }^{65}$ N. I. Geddes, ${ }^{65}$ G. P. Gopal, ${ }^{65}$ E. O. Olaiya, ${ }^{65}$

S. M. Xella, ${ }^{65}$ R. Aleksan, ${ }^{66}$ S. Emery, ${ }^{66}$ A. Gaidot, ${ }^{66}$ S. F. Ganzhur, ${ }^{66}$ P.-F. Giraud, ${ }^{66}$ G. Hamel de Monchenault, ${ }^{66}$ W. Kozanecki, ${ }^{66}$ M. Langer ${ }^{66}$ M. Legendre, ${ }^{66}$ G. W. London, ${ }^{66}$ B. Mayer, ${ }^{66}$ G. Schott, ${ }^{66}$ G. Vasseur ${ }^{66}$ Ch. Yèche, ${ }^{66}$

M. Zito, ${ }^{66}$ M. V. Purohit, ${ }^{67}$ A. W. Weidemann, ${ }^{67}$ F. X. Yumiceva, ${ }^{67}$ D. Aston, ${ }^{68}$ R. Bartoldus, ${ }^{68}$ N. Berger ${ }^{68}$

A. M. Boyarski, ${ }^{68}$ O. L. Buchmueller ${ }^{68}$ M. R. Convery, ${ }^{68}$ M. Cristinziani, ${ }^{68}$ G. De Nardo, ${ }^{68}$ D. Dong, ${ }^{68}$ J. Dorfan, ${ }^{68}$

D. Dujmic, ${ }^{68}$ W. Dunwoodie, ${ }^{68}$ E. E. Elsen, ${ }^{68}$ R. C. Field, ${ }^{68}$ T. Glanzman, ${ }^{68}$ S. J. Gowdy, ${ }^{68}$ T. Hadig, ${ }^{68}$ V. Halyo, ${ }^{68}$

T. Hryn'ova, ${ }^{68}$ W. R. Innes, ${ }^{68}$ M. H. Kelsey, ${ }^{68}$ P. Kim, ${ }^{68}$ M. L. Kocian, ${ }^{68}$ D. W. G. S. Leith, ${ }^{68}$ J. Libby, ${ }^{68}$

S. Luitz, ${ }^{68}$ V. Luth, ${ }^{68}$ H. L. Lynch, ${ }^{68}$ H. Marsiske, ${ }^{68}$ R. Messner, ${ }^{68}$ D. R. Muller, ${ }^{68}$ C. P. O'Grady, ${ }^{68}$ V. E. Ozcan, ${ }^{68}$

A. Perazzo, ${ }^{68}$ M. Perl, ${ }^{68}$ S. Petrak, ${ }^{68}$ B. N. Ratcliff, ${ }^{68}$ A. Roodman, ${ }^{68}$ A. A. Salnikov, ${ }^{68}$ R. H. Schindler, ${ }^{68}$ J. Schwiening, ${ }^{68}$ G. Simi, ${ }^{68}$ A. Snyder, ${ }^{68}$ A. Soha, ${ }^{68}$ J. Stelzer, ${ }^{68}$ D. Su, ${ }^{68}$ M. K. Sullivan, ${ }^{68}$ J. Va'vra, ${ }^{68}$ S. R. Wagner, ${ }^{68}$ M. Weaver, ${ }^{68}$ A. J. R. Weinstein, ${ }^{68}$ W. J. Wisniewski, ${ }^{68}$ M. Wittgen, ${ }^{68}$ D. H. Wright, ${ }^{68}$ C. C. Young, ${ }^{68}$ P. R. Burchat, ${ }^{69}$ A. J. Edwards, ${ }^{69}$ T. I. Meyer, ${ }^{69}$ B. A. Petersen, ${ }^{69}$ C. Roat, ${ }^{69}$ S. Ahmed, ${ }^{70}$ M. S. Alam, ${ }^{70}$ J. A. Ernst, ${ }^{70}$ M. A. Saeed, ${ }^{70}$ M. Saleem, ${ }^{70}$ F. R. Wappler, ${ }^{70}$ W. Bugg, ${ }^{71}$ M. Krishnamurthy, ${ }^{71}$ S. M. Spanier, ${ }^{71}$ R. Eckmann, ${ }^{72}$ H. Kim, ${ }^{72}$ J. L. Ritchie, ${ }^{72}$ A. Satpathy,${ }^{72}$ R. F. Schwitters, ${ }^{72}$ J. M. Izen, ${ }^{73}$

I. Kitayama, ${ }^{73}$ X. C. Lou, ${ }^{73}$ S. Ye, ${ }^{73}$ F. Bianchi, ${ }^{74}$ M. Bona,${ }^{74}$ F. Gallo, ${ }^{74}$ D. Gamba, ${ }^{74}$ C. Borean, ${ }^{75}$ L. Bosisio, ${ }^{75}$

F. Cossutti, ${ }^{75}$ G. Della Ricca, ${ }^{75}$ S. Dittongo, ${ }^{75}$ S. Grancagnolo, ${ }^{75}$ L. Lanceri, ${ }^{75}$ P. Poropat $,{ }^{75}, \ddagger$ L. Vitale, ${ }^{75}$

G. Vuagnin, ${ }^{75}$ R. S. Panvini, ${ }^{76}$ Sw. Banerjee, ${ }^{77}$ C. M. Brown, ${ }^{77}$ D. Fortin, ${ }^{77}$ P. D. Jackson, ${ }^{77}$ R. Kowalewski, ${ }^{77}$ J. M. Roney, ${ }^{77}$ H. R. Band, ${ }^{78}$ S. Dasu, ${ }^{78}$ M. Datta, ${ }^{78}$ A. M. Eichenbaum, ${ }^{78}$ J. J. Hollar, ${ }^{78}$ J. R. Johnson, ${ }^{78}$ P. E. Kutter, ${ }^{78}$ H. Li, ${ }^{78}$ R. Liu, ${ }^{78}$ F. Di Lodovico, ${ }^{78}$ A. Mihalyi, ${ }^{78}$ A. K. Mohapatra, ${ }^{78}$ Y. Pan, ${ }^{78}$ R. Prepost, ${ }^{78}$ S. J. Sekula, ${ }^{78}$ P. Tan, ${ }^{78}$ J. H. von Wimmersperg-Toeller, ${ }^{78}$ J. Wu, ${ }^{78}$ S. L. Wu, ${ }^{78}$ Z. Yu, ${ }^{78}$ and H. Neal ${ }^{79}$

(The BABAR Collaboration)

\author{
${ }^{1}$ Laboratoire de Physique des Particules, F-74941 Annecy-le-Vieux, France \\ ${ }^{2}$ Università di Bari, Dipartimento di Fisica and INFN, I-70126 Bari, Italy \\ ${ }^{3}$ Institute of High Energy Physics, Beijing 100039, China \\ ${ }^{4}$ University of Bergen, Inst. of Physics, N-500'7 Bergen, Norway \\ ${ }^{5}$ Lawrence Berkeley National Laboratory and University of California, Berkeley, CA 94720, USA \\ ${ }^{6}$ University of Birmingham, Birmingham, B15 2TT, United Kingdom \\ ${ }^{7}$ Ruhr Universität Bochum, Institut für Experimentalphysik 1, D-44780 Bochum, Germany \\ ${ }^{8}$ University of Bristol, Bristol BS8 1TL, United Kingdom \\ ${ }^{9}$ University of British Columbia, Vancouver, BC, Canada V6T $1 Z 1$ \\ ${ }^{10}$ Brunel University, Uxbridge, Middlesex UB8 3PH, United Kingdom \\ ${ }^{11}$ Budker Institute of Nuclear Physics, Novosibirsk 630090, Russia \\ ${ }^{12}$ University of California at Irvine, Irvine, CA 92697, USA \\ ${ }^{13}$ University of California at Los Angeles, Los Angeles, CA 90024, USA \\ ${ }^{14}$ University of California at Riverside, Riverside, CA 92521, USA \\ ${ }^{15}$ University of California at San Diego, La Jolla, CA 92093, USA \\ ${ }^{16}$ University of California at Santa Barbara, Santa Barbara, CA 93106, USA \\ ${ }^{17}$ University of California at Santa Cruz, Institute for Particle Physics, Santa Cruz, CA 95064, USA \\ ${ }^{18}$ California Institute of Technology, Pasadena, CA 91125, USA
}


${ }^{19}$ University of Cincinnati, Cincinnati, OH 45221, USA

${ }^{20}$ University of Colorado, Boulder, CO 80309, USA

${ }^{21}$ Colorado State University, Fort Collins, CO 80523, USA

${ }^{22}$ Technische Universität Dresden, Institut für Kern- und Teilchenphysik, D-01062 Dresden, Germany

${ }^{23}$ Ecole Polytechnique, LLR, F-91128 Palaiseau, France

${ }^{24}$ University of Edinburgh, Edinburgh EH9 3JZ, United Kingdom

${ }^{25}$ Università di Ferrara, Dipartimento di Fisica and INFN, I-44100 Ferrara, Italy

${ }^{26}$ Florida AEM University, Tallahassee, FL 3230\%, USA

${ }^{27}$ Laboratori Nazionali di Frascati dell'INFN, I-00044 Frascati, Italy

${ }^{28}$ Università di Genova, Dipartimento di Fisica and INFN, I-16146 Genova, Italy

${ }^{29}$ Harvard University, Cambridge, MA 02138, USA

${ }^{30}$ Universität Heidelberg, Physikalisches Institut, Philosophenweg 12, D-69120 Heidelberg, Germany

${ }^{31}$ Imperial College London, London, SW7 2AZ, United Kingdom

${ }^{32}$ University of Iowa, Iowa City, IA 52242, USA

${ }^{33}$ Iowa State University, Ames, IA 50011-3160, USA

${ }^{34}$ Laboratoire de l'Accélérateur Linéaire, F-91898 Orsay, France

${ }^{35}$ Lawrence Livermore National Laboratory, Livermore, CA 94550, USA

${ }^{36}$ University of Liverpool, Liverpool L69 72E, United Kingdom

${ }^{37}$ Queen Mary, University of London, E1 4NS, United Kingdom

${ }^{38}$ University of London, Royal Holloway and Bedford New College, Egham, Surrey TW20 OEX, United Kingdom

${ }^{39}$ University of Louisville, Louisville, KY 40292, USA

${ }^{40}$ University of Manchester, Manchester M13 9PL, United Kingdom

${ }^{41}$ University of Maryland, College Park, MD 20742, USA

${ }^{42}$ University of Massachusetts, Amherst, MA 01003, USA

${ }^{43}$ Massachusetts Institute of Technology, Laboratory for Nuclear Science, Cambridge, MA 02139, USA

${ }^{44}$ McGill University, Montréal, QC, Canada H3A $2 T 8$

${ }^{45}$ Università di Milano, Dipartimento di Fisica and INFN, I-20133 Milano, Italy

${ }^{46}$ University of Mississippi, University, MS 38677, USA

${ }^{47}$ Université de Montréal, Laboratoire René J. A. Lévesque, Montréal, QC, Canada H3C 3J7

${ }^{48}$ Mount Holyoke College, South Hadley, MA 01075, USA

${ }^{49}$ Università di Napoli Federico II, Dipartimento di Scienze Fisiche and INFN, I-80126, Napoli, Italy

${ }^{50}$ NIKHEF, National Institute for Nuclear Physics and High Energy Physics, NL-1009 DB Amsterdam, The Netherlands

${ }^{51}$ University of Notre Dame, Notre Dame, IN 46556, USA

${ }^{52}$ Oak Ridge National Laboratory, Oak Ridge, TN 37831, USA

${ }^{53}$ Ohio State University, Columbus, OH 43210, USA

${ }^{54}$ University of Oregon, Eugene, OR 97403, USA

${ }^{55}$ Università di Padova, Dipartimento di Fisica and INFN, I-35131 Padova, Italy

${ }^{56}$ Universités Paris VI et VII, Lab de Physique Nucléaire H. E., F-75252 Paris, France

${ }^{57}$ Università di Pavia, Dipartimento di Elettronica and INFN, I-27100 Pavia, Italy

${ }^{58}$ University of Pennsylvania, Philadelphia, PA 19104, USA

${ }^{59}$ Università di Perugia, Dipartimento di Fisica and INFN, I-06100 Perugia, Italy

${ }^{60}$ Università di Pisa, Dipartimento di Fisica, Scuola Normale Superiore and INFN, I-56127 Pisa, Italy

${ }^{61}$ Prairie View A\&M University, Prairie View, TX 77446, USA

${ }^{62}$ Princeton University, Princeton, NJ 08544, USA

${ }^{63}$ Università di Roma La Sapienza, Dipartimento di Fisica and INFN, I-00185 Roma, Italy

${ }^{64}$ Universität Rostock, D-18051 Rostock, Germany

${ }^{65}$ Rutherford Appleton Laboratory, Chilton, Didcot, Oxon, OX11 0QX, United Kingdom

${ }^{66}$ DSM/Dapnia, CEA/Saclay, F-91191 Gif-sur-Yvette, France

${ }^{67}$ University of South Carolina, Columbia, SC 29208, USA

${ }^{68}$ Stanford Linear Accelerator Center, Stanford, CA 94309, USA

${ }^{69}$ Stanford University, Stanford, CA 94305-4060, USA

${ }^{70}$ State Univ. of New York, Albany, NY 12222, USA

${ }^{71}$ University of Tennessee, Knoxville, TN 37996, USA

${ }^{72}$ University of Texas at Austin, Austin, TX 78712, USA

${ }^{73}$ University of Texas at Dallas, Richardson, TX 75083, USA

${ }^{74}$ Università di Torino, Dipartimento di Fisica Sperimentale and INFN, I-10125 Torino, Italy

${ }^{75}$ Università di Trieste, Dipartimento di Fisica and INFN, I-34127 Trieste, Italy

${ }^{76}$ Vanderbilt University, Nashville, TN 37235, USA

${ }^{77}$ University of Victoria, Victoria, BC, Canada V8W $3 P 6$

${ }^{78}$ University of Wisconsin, Madison, WI 53706, USA

${ }^{79}$ Yale University, New Haven, CT 06511, USA

(Dated: December 8, 2003) 


\begin{abstract}
A search for the lepton-flavor-violating decay of the tau into three charged leptons has been performed using $91.5 \mathrm{fb}^{-1}$ of data collected at an $e^{+} e^{-}$center-of-mass energy of $10.58 \mathrm{GeV}$ with the BABAR detector at the PEP-II storage ring. In all six decay modes considered, the numbers of events found in data are compatible with the background expectations. Upper limits on the branching fractions are set in the range $(1-3) \times 10^{-7}$ at $90 \%$ confidence level.
\end{abstract}

PACS numbers: 13.35.Dx, 14.60.Fg, 11.30.Hv

Lepton-flavor violation (LFV) involving charged leptons has never been observed, and stringent experimental limits exist from muon branching fractions: $\mathcal{B}(\mu \rightarrow e \gamma)<$ $1.2 \times 10^{-11}[1]$ and $\mathcal{B}(\mu \rightarrow e e e)<1.0 \times 10^{-12}[2]$ at $90 \%$ confidence level (CL). Recent results from neutrino oscillation experiments [3] show that LFV does indeed occur, although the branching fractions expected in charged lepton decay due to neutrino mixing alone are probably no more than $10^{-14}[4]$.

In tau decays, the most stringent limit on LFV is $\mathcal{B}(\tau \rightarrow \mu \gamma)<3.1 \times 10^{-7}$ at $90 \%$ CL [5]. Many extensions to the Standard Model (SM), particularly models seeking to describe neutrino mixing, predict enhanced LFV in tau decays over muon decays with branching fractions from $10^{-10}$ up to the current experimental limits [6]. Observation of LFV in tau decays would be a clear signature of non-SM physics, while improved limits will provide further constraints on theoretical models.

This analysis is based on data recorded by the BABAR detector at the PEP-II asymmetric-energy $e^{+} e^{-}$storage ring operated at the Stanford Linear Accelerator Center. The data sample consists of $81.9 \mathrm{fb}^{-1}$ recorded at $\sqrt{s}=$ $10.58 \mathrm{GeV}$ and $9.6 \mathrm{fb}^{-1}$ recorded at $\sqrt{s}=10.54 \mathrm{GeV}$. With an expected cross section for tau pairs at the luminosity-weighted $\sqrt{s}$ of $\sigma_{\tau \tau}=(0.89 \pm 0.02) \mathrm{nb}$ [7], this data sample contains over 160 million tau decays.

The BABAR detector is described in detail in Ref. [8]. Charged-particle (track) momenta are measured with a 5-layer double-sided silicon vertex tracker and a 40-layer drift chamber inside a 1.5-T superconducting solenoidal magnet. The transverse momentum resolution parameterized as $\sigma_{p_{T}} / p_{T}=\left(0.13 \cdot p_{T} /[\mathrm{GeV} / c]+0.45\right) \%$ is achieved. An electromagnetic calorimeter consisting of $6580 \mathrm{CsI}(\mathrm{Tl})$ crystals is used to identify electrons and photons, a ring-imaging Cherenkov detector is used to identify charged hadrons, and the instrumented magnetic flux return (IFR) is used to identify muons. Particle attributes are reconstructed in the laboratory frame and then boosted to the $e^{+} e^{-}$center-of-mass (CM) frame using the measured asymmetric beam energies.

This paper presents a search for LFV in the neutrinoless decay $\tau^{-} \rightarrow \ell^{-} \ell^{+} \ell^{-}$. All possible lepton combinations consistent with charge conservation are considered, leading to six distinct decay modes $\left(e^{-} e^{+} e^{-}, \mu^{+} e^{-} e^{-}\right.$, $\mu^{-} e^{+} e^{-}, e^{+} \mu^{-} \mu^{-}, e^{-} \mu^{+} \mu^{-}, \mu^{-} \mu^{+} \mu^{-}$) [9]. The signature of this process is three charged particles, each identified as either an electron or muon, with an invariant mass and energy equal to that of the parent tau lep- ton. Candidate signal events in this analysis are required to have a "1-3 topology," where one tau decay yields three charged particles (3-prong), while the second tau decay yields one charged particle (1-prong). Four well reconstructed tracks are required with zero net charge, pointing towards a common region consistent with $\tau^{+} \tau^{-}$ production and decay. One of these tracks must be separated from the other three by at least $90^{\circ}$ in the $\mathrm{CM}$ frame. The plane perpendicular to this isolated track divides the event into two hemispheres and defines the 1-3 topology. Pairs of oppositely charged tracks identified as photon conversions in the detector material with an $e^{+} e^{-}$invariant mass below $30 \mathrm{MeV} / c^{2}$ are ignored.

Each of the charged particles found in the 3-prong hemisphere must be identified as either an electron or muon candidate. Electrons are identified using the ratio of calorimeter energy to track momentum $(E / p)$, the ionization loss in the tracking system $(\mathrm{d} E / \mathrm{d} x)$, and the shape of the shower in the calorimeter. Muons are identified by hits in the IFR and small energy deposits in the calorimeter. Muons with momentum less than $0.5 \mathrm{GeV} / c$ cannot be identified because they do not penetrate far enough into the IFR.

The particle identification (PID) requirements are not sufficient to suppress certain backgrounds, particularly those from higher-order radiative Bhabha and $\mu^{+} \mu^{-}$ events that can have four leptons in the final state. To reduce these backgrounds, additional selection criteria are applied to the six different decay modes. For all decay modes, the momentum of the 1-prong track is required to be less than $4.8 \mathrm{GeV} / \mathrm{c}$ in the $\mathrm{CM}$ frame. For the $e^{-} e^{+} e^{-}$and $e^{-} \mu^{+} \mu^{-}$decay modes, the charged particle in the 1-prong hemisphere must not be identified as an electron, while for the $\mu^{-} e^{+} e^{-}$and $\mu^{-} \mu^{+} \mu^{-}$decay modes it must not be a muon. For all four of these decay modes, the angle $\theta_{13}$ between the 1-prong momentum and the vector sum of the 3-prong momenta in the CM frame must satisfy $\cos \theta_{13}>-0.9999$, while the net transverse momentum of the four tracks must be greater than $0.1 \mathrm{GeV} / c$. Additional requirements are imposed to reduce the $q \bar{q}$ and $\mathrm{SM} \tau^{+} \tau^{-}$backgrounds. Events in the four decay modes specified above are required to have no unassociated calorimeter clusters (photons) in the 3prong hemisphere with energy greater than $100 \mathrm{MeV}$ in the laboratory frame, while events in all six decay modes are required to have no track in the 3-prong hemisphere that is also consistent with being a kaon.

To reduce backgrounds further, signal events are re- 
quired to have an invariant mass and total energy in the 3 -prong hemisphere consistent with a parent tau lepton. These quantities are calculated from the observed track momenta assuming the corresponding lepton masses for each decay mode. The energy difference is defined as $\Delta E \equiv E_{\text {rec }}^{\star}-E_{\text {beam }}^{\star}$, where $E_{\text {rec }}^{\star}$ is the total energy of the tracks observed in the 3-prong hemisphere and $E_{\text {beam }}^{\star}$ is the beam energy, both in the CM frame. The mass difference is defined as $\Delta M \equiv M_{\text {rec }}-m_{\tau}$ where $M_{\text {rec }}$ is the reconstructed invariant mass of the three tracks and $m_{\tau}=1.777 \mathrm{GeV} / c^{2}$ is the tau mass [10].

The signal distributions in the $(\Delta M, \Delta E)$ plane are broadened by detector resolution and radiative effects. The radiation of photons from the incoming $e^{+} e^{-}$particles before annihilation affects all decay modes, leading to a tail at low values of $\Delta E$. Radiation from the finalstate leptons is more likely for electrons than muons, and produces a tail at low values of $\Delta M$ as well. Rectangular signal regions are defined separately for each decay mode as follows. For all six decay modes, the upper right corner of the signal region is fixed at $\left(30 \mathrm{MeV} / c^{2}, 50 \mathrm{MeV}\right)$, while the lower left corner is at $(-70,-120)$ for the $e^{-} e^{+} e^{-}$ and $\mu^{-} e^{+} e^{-}$decay modes, $(-100,-200)$ for $\mu^{+} e^{-} e^{-}$, $(-50,-200)$ for $e^{+} \mu^{-} \mu^{-},(-50,-150)$ for $e^{-} \mu^{+} \mu^{-}$, and $(-30,-150)$ for $\mu^{-} \mu^{+} \mu^{-}$. All values are given in units of $\left(\mathrm{MeV} / \mathrm{c}^{2}, \mathrm{MeV}\right)$. These signal region boundaries are chosen to provide the smallest expected upper limits on the branching fractions in the background-only hypothesis. These expected upper limits are estimated using only Monte Carlo (MC) simulations and data control samples, not candidate signal events. Figure 1 shows the observed data in the $(\Delta M, \Delta E)$ plane, along with the signal region boundaries and the expected signal distributions. To avoid bias, a blinded analysis procedure was adopted with the number of data events in the signal region remaining unknown until the selection criteria were finalized and all cross checks were performed.

The efficiency of the selection for signal events is estimated with a MC simulation of LFV tau decays. Simulated tau-pair events including higher-order radiative corrections are generated using KK2f [7] with one tau decaying to three leptons with a 3-body phase space distribution, while the other tau decays according to measured rates [11] simulated with Tauola [12]. Final state radiative effects are simulated for all decays using Photos [13]. The detector response is simulated with GEANT4 [14], and the simulated events are then reconstructed in the same manner as data.

About $50 \%$ of the MC signal events pass the 1-3 topology requirement. The lepton identification efficiencies and misidentification probabilities are measured using tracks in kinematically-selected data samples (radiative Bhabha, radiative $\mu^{+} \mu^{-}$, two-photon $e^{+} e^{-} \ell^{+} \ell^{-}$, and $J / \psi \rightarrow \ell^{+} \ell^{-}$) and parameterized as a function of particle momentum, polar angle, and azimuthal angle in the laboratory frame. These data-derived efficiencies are then

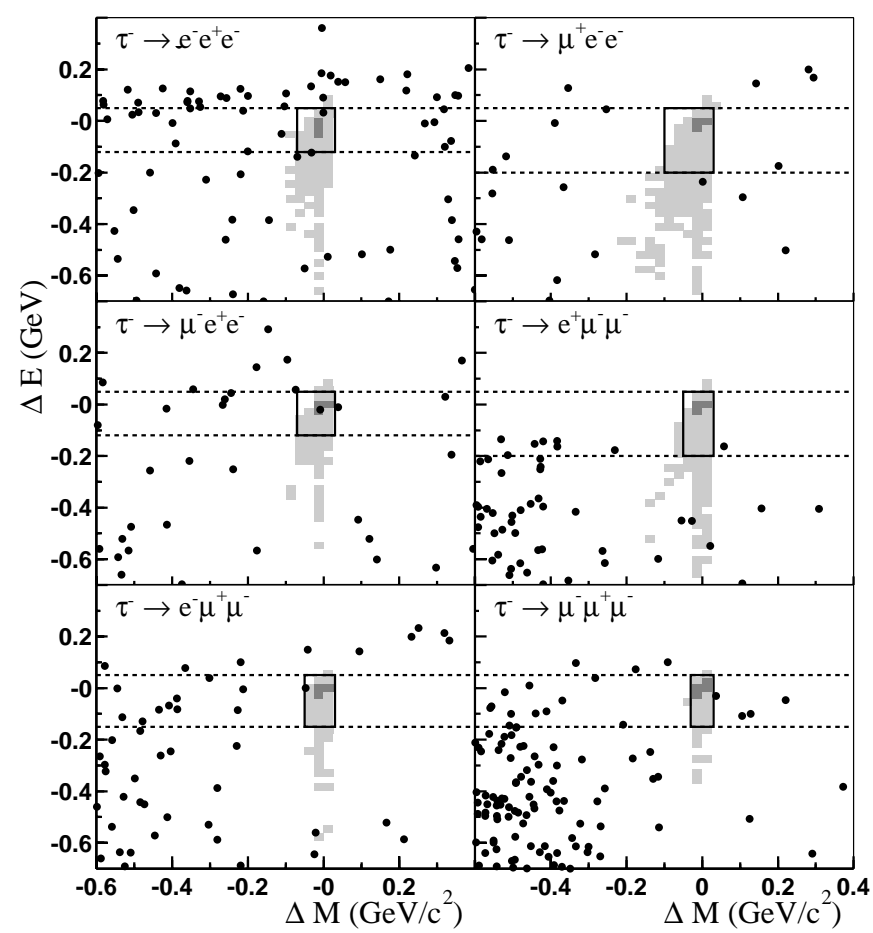

FIG. 1: Observed data shown as dots in the $(\Delta M, \Delta E)$ plane and the boundaries of the signal region for each decay mode. The dark and light shading indicates contours containing $50 \%$ and $90 \%$ of the selected MC signal events, respectively. The regions shown in Fig. 2 are indicated by dashed lines.

used to give the probability that a simulated MC particle will be identified (or misidentified) as an electron or a muon. For the lepton momentum spectrum predicted by the signal MC, the electron and muon identification requirements are found to have an average efficiency per lepton of $91 \%$ and $63 \%$, respectively. The probability for a hadron to be misidentified as an electron in SM 3 -prong tau decays is $2.2 \%$, while the probability to be misidentified as a muon is $4.8 \%$ [15]. The final efficiency for signal events to be found in the signal region is shown in Table I for each decay mode and ranges from $7 \%$ to $12 \%$. This efficiency includes the $85 \%$ branching fraction for 1-prong tau decays.

There are three main classes of background remaining after the selection criteria are applied: low multiplicity $q \bar{q}$ events (mainly continuum light-quark production), QED events (Bhabha and $\mu^{+} \mu^{-}$), and SM $\tau^{+} \tau^{-}$events. These three background classes have distinctive distributions in the $(\Delta M, \Delta E)$ plane: $q \bar{q}$ events tend to populate the plane uniformly, while QED backgrounds are restricted to a narrow band at positive values of $\Delta E$, and $\tau^{+} \tau^{-}$ backgrounds are restricted to negative values of both $\Delta E$ and $\Delta M$. A negligible two-photon background remains.

The expected background rates for each decay mode are determined by fitting a set of probability density functions (PDFs) to the observed data in the $(\Delta M, \Delta E)$ 
TABLE I: Efficiency estimates, number of expected background events $\left(N_{\text {bgd }}\right)$, number of observed events $\left(N_{\text {obs }}\right)$, and branching fraction upper limits for each decay mode.

\begin{tabular}{lccc}
\hline \hline Decay mode & $e^{-} e^{+} e^{-}$ & $\mu^{+} e^{-} e^{-}$ & $\mu^{-} e^{+} e^{-}$ \\
\hline Efficiency [\%] & $7.3 \pm 0.2$ & $11.6 \pm 0.4$ & $7.7 \pm 0.3$ \\
\hline$q \bar{q}$ bgd. & 0.67 & 0.17 & 0.39 \\
QED bgd. & 0.84 & 0.20 & 0.23 \\
$\tau^{+} \tau^{-}$bgd. & 0.00 & 0.01 & 0.00 \\
\hline$N_{\text {bgd }}$ & $1.51 \pm 0.11$ & $0.37 \pm 0.08$ & $0.62 \pm 0.10$ \\
$N_{\text {obs }}$ & 1 & 0 & 1 \\
\hline $\mathcal{B}_{\mathrm{UL}}^{90}$ & $2.0 \times 10^{-7}$ & $1.1 \times 10^{-7}$ & $2.7 \times 10^{-7}$ \\
\hline \hline Decay mode & $e^{+} \mu^{-} \mu^{-}$ & $e^{-} \mu^{+} \mu^{-}$ & $\mu^{-} \mu^{+} \mu^{-}$ \\
\hline Efficiency [\%] & $9.8 \pm 0.5$ & $6.8 \pm 0.4$ & $6.7 \pm 0.5$ \\
\hline$q \bar{q}$ bgd. & 0.20 & 0.19 & 0.29 \\
QED bgd. & 0.00 & 0.19 & 0.01 \\
$\tau^{+} \tau^{-}$bgd. & 0.01 & 0.01 & 0.01 \\
\hline$N_{\text {bgd }}$ & $0.21 \pm 0.07$ & $0.39 \pm 0.08$ & $0.31 \pm 0.09$ \\
$N_{\text {obs }}$ & 0 & 1 & 0 \\
\hline $\mathcal{B}_{\mathrm{UL}}^{90}$ & $1.3 \times 10^{-7}$ & $3.3 \times 10^{-7}$ & $1.9 \times 10^{-7}$ \\
\hline \hline
\end{tabular}

plane in a grand sideband (GS) region. The GS region, shown in Fig. 1, is defined as the rectangle bounded by the points $\left(-600 \mathrm{MeV} / c^{2},-700 \mathrm{MeV}\right)$ and $\left(400 \mathrm{MeV} / c^{2}, 400 \mathrm{MeV}\right)$, excluding the signal region. For both the $q \bar{q}$ and $\tau^{+} \tau^{-}$backgrounds, an analytic PDF is constructed from the product of two PDFs $P_{M}$ and $P_{E}$, where $P_{M}(\Delta M)$ is the sum of two Gaussians with a common mean and $P_{E}(\Delta E)=\left(1-x / \sqrt{1+x^{2}}\right)\left(1+a x+b x^{2}+\right.$ $\left.c x^{3}\right)$ with $x=(\Delta E-d) / e[16]$. The shapes of these PDFs are described by a total of nine free parameters, which are determined by fits to MC $q \bar{q}$ and $\tau^{+} \tau^{-}$background samples for each decay mode.

For the QED backgrounds, an analytic PDF is constructed from the product of a Crystal Ball function [17] in $\Delta E^{\prime}$ and a linear function in $\Delta M^{\prime}$, where the $\left(\Delta M^{\prime}, \Delta E^{\prime}\right)$ axes have been rotated slightly from $(\Delta M, \Delta E)$ to fit the observed distribution. The six parameters of this $\mathrm{PDF}$, including the rotation angle, are obtained by fitting control samples with a 1-3 topology that are enhanced in Bhabha or $\mu^{+} \mu^{-}$events by requiring that the particle in the 1-prong hemisphere is identified as an electron or muon. Any value for $\cos \theta_{13}$ is allowed, but the control sample events otherwise pass the selection criteria.

With the shapes of the three background PDFs determined, an unbinned maximum likelihood fit to the data in the GS region is used to find the expected rate of each background type in the signal region, as shown in Table I. The PDF shape determinations and background fits are performed separately for each of the six decay modes. Figure 2 shows the data and the background PDFs for values of $\Delta E$ in the signal range.

The largest systematic uncertainty in the signal efficiency is due to the uncertainty in measuring the PID efficiencies. This uncertainty is determined from the sta-

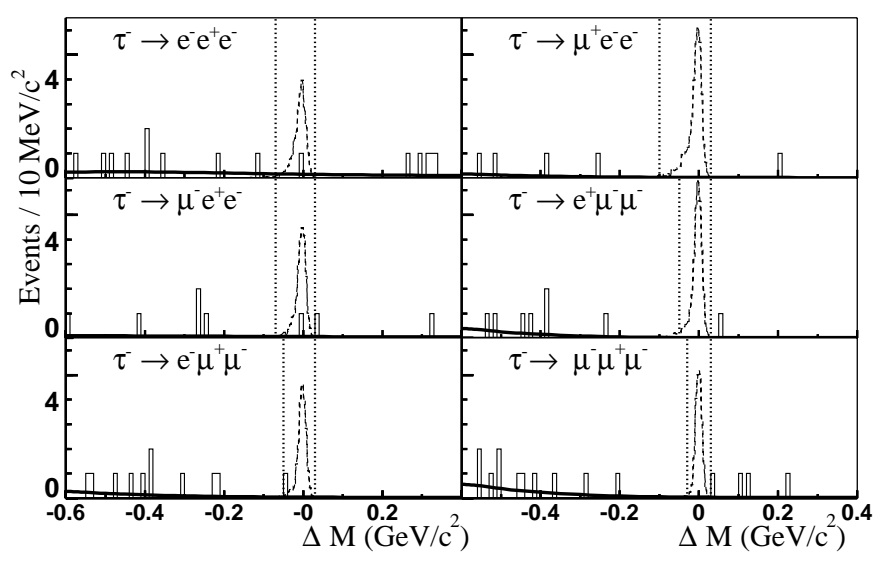

FIG. 2: Distribution of $\Delta M$ for data (solid histogram) and background PDFs (solid curves) for events with $\Delta E$ in the signal region indicated in Fig. 1. Expected signal distributions are shown (dashed histogram) for a branching fraction of $10^{-6}$.

tistical precision of the PID control samples, and ranges from $0.7 \%$ for $e^{-} e^{+} e^{-}$to $6.2 \%$ for $\mu^{-} \mu^{+} \mu^{-}$relative to the efficiency [18]. The modeling of the tracking efficiency contributes an additional $2 \%$ uncertainty, as does the statistical limitation of the MC signal sample. All other sources of uncertainty are found to be small, including the modeling in the generator of radiative effects, track momentum resolution, trigger performance, observables used in the selection criteria, and knowledge of the tau 1-prong branching fractions. The efficiency has been estimated using a 3-body phase space model and no uncertainty is assigned for possible model dependence. The selection efficiency is found to be uniform within $10 \%$ across the Dalitz plane, provided the invariant mass for any pair of leptons is less than $1.4 \mathrm{GeV} / c^{2}$.

Since the background levels are extracted directly from the data, systematic uncertainties on the background estimation are directly related to the background parameterization and the fit technique used. The finite data available in the GS region to determine the background rates is the largest uncertainty and varies from $10 \%$ to $25 \%$ depending upon the decay mode. Additional uncertainties are estimated by varying the fit procedure and changing the functional form of the background PDFs. Cross checks of the background estimation were performed by considering the number of events expected and observed in sideband regions immediately neighboring the signal region for each decay mode.

The numbers of events observed $\left(N_{\mathrm{obs}}\right)$ and the background expectations $\left(N_{\mathrm{bgd}}\right)$ are shown in Table I, with no significant excess found in any decay mode. Upper limits on the branching fractions are calculated according to $\mathcal{B}_{\mathrm{UL}}^{90}=N_{\mathrm{UL}}^{90} /\left(2 \varepsilon \mathcal{L} \sigma_{\tau \tau}\right)$, where $N_{\mathrm{UL}}^{90}$ is the $90 \%$ CL upper limit for the number of signal events when $N_{\text {obs }}$ events are observed with $N_{\mathrm{bgd}}$ background events expected. The values $\varepsilon, \mathcal{L}$, and $\sigma_{\tau \tau}$ are the selection efficiency, luminos- 
ity, and $\tau^{+} \tau^{-}$cross section, respectively. The estimates of $\mathcal{L}=91.5 \mathrm{fb}^{-1}$ and $\sigma_{\tau \tau}=0.89 \mathrm{nb}$ are correlated [19], and the uncertainty on the product $\mathcal{L} \sigma_{\tau \tau}$ is $2.3 \%$. The branching fraction upper limits have been calculated including all uncertainties using the technique of Cousins and Highland [20] following the implementation of Barlow [21]. The 90\% CL upper limits on the $\tau^{-} \rightarrow \ell^{-} \ell^{+} \ell^{-}$ branching fractions, shown in Table I, are in the range $(1-3) \times 10^{-7}$.

We are grateful for the excellent luminosity and machine conditions provided by our PEP-II colleagues, and for the substantial dedicated effort from the computing organizations that support BABAR. The collaborating institutions wish to thank SLAC for its support and kind hospitality. This work is supported by DOE and NSF (USA), NSERC (Canada), IHEP (China), CEA and CNRS-IN2P3 (France), BMBF and DFG (Germany), INFN (Italy), FOM (The Netherlands), NFR (Norway), MIST (Russia), and PPARC (United Kingdom). Individuals have received support from the A. P. Sloan Foundation, Research Corporation, and Alexander von Humboldt Foundation.

* Also with Università della Basilicata, Potenza, Italy

† Also with IFIC, Instituto de Física Corpuscular, CSICUniversidad de Valencia, Valencia, Spain

$\ddagger$ Deceased

[1] MEGA/LAMPF Collaboration, M. L. Brooks et al., Phys. Rev. Lett. 83, 1521 (1999).

[2] SINDRUM Collaboration, U. Bellgardt et al., Nucl. Phys. B 299, 1 (1998).

[3] K2K Collaboration, M. H. Ahn et al., Phys. Rev. Lett. 90, 041801 (2003); KamLAND Collaboration, K. Eguchi et al., Phys. Rev. Lett. 90, 021802 (2003); SNO Collaboration, Q. R. Ahmad et al., Phys. Rev. Lett. 89, 011301 (2002); Super-Kamiokande Collaboration, Y. Fukuda et al., Phys. Rev. Lett. 81, 1562 (1998).

[4] X. Y. Pham, Eur. Phys. Jour. C 8, 513 (1999).

[5] Belle Collaboration, K. Abe et al., hep-ex/0310029.

[6] E. Ma, Nucl. Phys. B Proc. Suppl. 123, 125 (2003).

[7] B. F. Ward, S. Jadach, and Z. Was, Nucl. Phys. Proc. Suppl. 116, 73 (2003).

[8] BABAR Collaboration, B. Aubert et al., Nucl. Instr. Meth. A 479, 1 (2002).

[9] Throughout this paper, charge conjugate decay modes also are implied.

[10] BES Collaboration, J. Z. Bai et al., Phys. Rev. D 53, 20 (1996).

[11] Particle Data Group, K. Hagiwara et al., Phys. Rev. D 66, 010001 (2002).

[12] S. Jadach, Z. Was, R. Decker, and J. H. Kuhn, Comput. Phys. Commun. 76, 361 (1993).

[13] E. Barberio and Z. Was, Comput. Phys. Commun. 79, 291 (1994).

[14] GEANT4 Collaboration, S. Agostinelli et al., Nucl. Instr. Meth. A 506, 250 (2003).

[15] Due to the need to identify three leptons, the PID requirements emphasize efficiency over purity, resulting in a relatively high misidentification rate.

[16] OPAL Collaboration, G. Abbiendi et al., Phys. Lett. B 492, 23 (2000).

[17] Crystal Ball Collaboration, T. Skwarnicki, DESY-F3186-02 (1986).

[18] All uncertainties quoted in the text are relative.

[19] The luminosity is measured using the observed $\mu^{+} \mu^{-}$ rate, and the $\mu^{+} \mu^{-}$and $\tau^{+} \tau^{-}$cross sections are both estimated with KK2f.

[20] R. D. Cousins and V. L. Highland, Nucl. Instrum. Meth. A 320, 331 (1992).

[21] R. Barlow, Comput. Phys. Commun. 149, 97 (2002). 